\title{
Modelica Spur Gears with Hertzian Contact Forces
}

\author{
Markus Dahl ${ }^{1}$ Håkan Wettergren ${ }^{1}$ Henrik Tidefelt ${ }^{1}$ \\ ${ }^{1}$ Wolfram MathCore, Linköping, Sweden, \{markusd, hakanw, henrikt\}@wolfram.com
}

\begin{abstract}
To be able to capture the dynamics of entire systems is one of the strengths of the Modelica language. This article will examine the possibility of modeling spur gears in the Modelica environment Wolfram SystemModeler, and integrating them with other rotating machinery elements, such as roller bearings and flexible shafts. The contact forces between spur gears are based on the Hertzian Theory of Contact ${ }^{1}$

Keywords: Spur Gear, Hertz Contact Theory, Rotating Machinery
\end{abstract}

\section{Introduction}

Gear contact forces can be accurately modelled by FEM programs, but usually at a high computational cost. The focus is usually on solving the force equations statically, where some dynamics might be lost. A common way of calculating the dynamics is to add a so called application factor to the static solution, approximating the dynamic result. By using a Modelica model instead, the dynamics can be included in the model, replacing the application factor. To be compatible with other libraries, the models here are based on the MultiBody library from the Modelica Standard Library. The choice of using a 3D mechanical library instead of libraries such as PlanarMechanics (Zimmer, 2012), is to be able to keep building on these models to handle helical and other type of gears.

Another benefit of Modelica models is that they can be used with other rotating machinery elements. Let's say that a wind turbine gear box should be analyzed. This gear box contains inner and outer spur gears, flexible shafts, and roller bearings. If a Modelica model is used for this purpose, we can see both how the spur gears affect the bearings, the shafts, as well as how the shafts and bearings affect the gears. Therefore, the dynamics of the entire system can be captured and analyzed.

In Section 2, the gear geometry of a spur gear will be introduced. Following that, Section 3 will be an introduction to the Hertzian Contact theory. Section 4 will explain how this was implemented in Modelica. Section 5 will go through some examples where the gears were used. Finally a discussion of the results will follow in Section 6.

${ }^{1}$ As found in Roark's Formulas for Stress and Strain, 2002

\subsection{Previous work}

Several papers have been written regarding modeling gears in the Modelica language. Special attention has been seen in the area of powertrains. One of the original papers is (Otter et al., 2000) where the PowerTrain package was presented.

The package PowerTrains contains 1-dimensional, rotational mechanical systems. I.e. a lot of simplifications have been made to be able to model the complete driveline. At that time it was a state of the art approach. However, the very idealized components in that library cannot be used for any advanced dimensioning or root cause analysis. The description of the components are very simplified compared to the special simulation tools that exist in each specific machine element area.

During the years, several of the components have been refined. One work is (van der Linden and de Souza Silva, 2009) where a 3-Dof elastic model was used which included the elasticity of the support bearings in the load direction, which was not possible in the standard gear model. The model was then extended in (van der Linden, 2012) to also include a Gear Contact Model. A later paper, (van der Linden, 2015) compared the results from a Modelica model that investigated gear contact to tests.

A much more detailed approach was taken by (Kosenko and Gusev, 2011) and further improved in (Kosenko and Gusev, 2012), where the forces between gears were modelled with high detail in a Modelica environment.

\section{Gear Geometry}

This section describes the geometrical modeling of two gear wheels that are in contact or in close proximity. Starting with the geometry of a single gear wheel, we then proceed with the geometry of the interaction between two gear wheels, before going into more advanced topics such as tip relief and the geometry involved in triggering events in a Modelica model.

\subsection{Geometry of a gear wheel}

A gear is basically a toothed wheel aimed to transmit rotation from one shaft to another. Spur gears, that is the focus in this article, can be described as parallelaxes gears without a helical angle. The gears can be of 

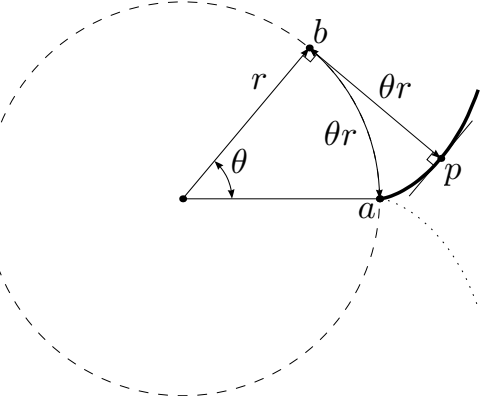

Figure 1. Involute of a circle. The involute (thick line) is traced by mapping each angle $\theta$ to the point $p$ by going the distance $\theta r$ along the circle from point $a$ to point $b$, and then going the same distance back along the tangent to point $p$. Note the right angle at point $p$. The involute obtained by this procedure creates an involute corresponding to a rear flank of a gear tooth. Mirroring the procedure creates a front flank, drawn with a dotted line.

Table 1. Gear wheel geometry parameters.

\begin{tabular}{ll}
\hline Parameter & Description \\
\hline$z$ & Number of teeth in gear \\
$m$ & Gear module \\
$\alpha_{0}=20^{\circ}$ & Reference profile angle \\
$x$ & Profile shift factor \\
\hline
\end{tabular}

two types, inner gear (a ring with teeth on the inside) and outer gear (a wheel with teeth on the outside. For clarity of presentation, only outer gears are considered in this section.

One of the main reasons for the broad use of gears is the efficiency of the transmission, which depends on shape of the teeth. The most common shape of a tooth is a circle involute. A circle involute or simply involute, is a curve following the end point of a tangent that is rolled up from a circle. It is defined by the geometry in Figure 1. The right angle at point $p$, between the tangent of the circle and the tangent
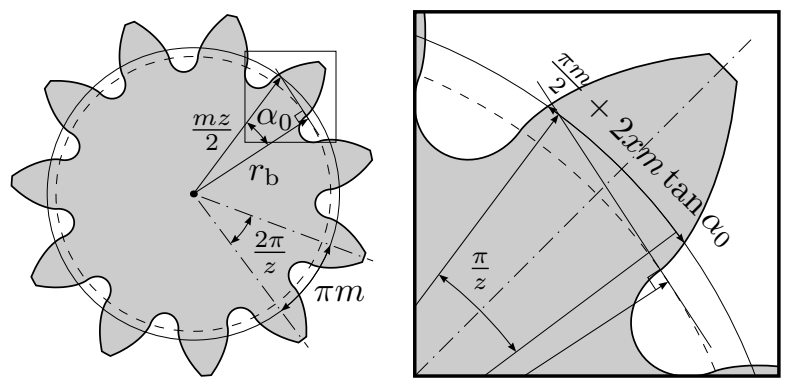

Figure 2. Gear wheel geometry parameters in an outer spur gear. The radius of the base circle, $r_{\mathrm{b}}$ is easily derived from the gear wheel parameters $z, m$, and $\alpha_{0}$. The effect of the profile shift, $x$, is best understood in relation to a straight gear reference profile, but the derivation is out of scope in the current presentation.

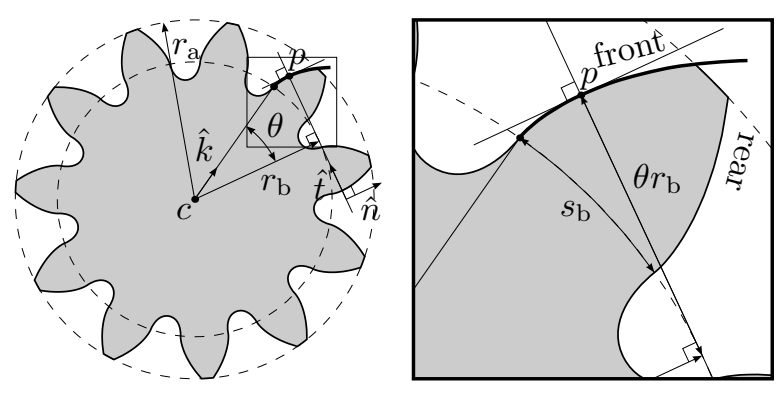

Figure 3. Intersection between gear flank and a tangent of the base circle. Also showing the derived quantity $s_{\mathrm{b}}$, and the additional parameter $r_{\mathrm{a}}$. Vector $\hat{k}$ points to the point on the tooth where the involute begins. The point $p$ is then obtained from the rolling angle, $\theta$, and $r_{\mathrm{b}}$. The front flank is where force is transmitted when applying torque in counter-clockwise direction, and the rear flank when applying clockwise torque.)

of the circle involute, is a fundamental property of involute curves.

There are four geometrical parameters that need to be specified for a spur gear in our case, listed in Table 1. These parameters are shown in Figure 2. From these user-specified parameters, many other quantities are derived, see Figure 3. For example, one can derive the tooth base thickness, $s_{\mathrm{b}}$, and also express a standard choice of $r_{\mathrm{a}}$, as

$$
\begin{aligned}
& s_{\mathrm{b}}=\left(\frac{\pi}{2}+2 x \tan \alpha_{0}+z \operatorname{inv} \alpha_{0}\right) m \cos \alpha_{0} \\
& r_{\mathrm{a}}=m\left(\frac{z}{2}+x+1\right)
\end{aligned}
$$

where inv $\alpha_{0}=\tan \alpha_{0}-\alpha_{0}$.

The signed rolling angle, $\theta$, is related to the inner product of the unit vectors $\hat{k}$ and $\hat{t}$,

$$
<\hat{k}, \hat{t}>=\cos \left(\frac{\pi}{2}-\theta\right)
$$

from which it can be solved reliably.

\subsection{Line of contact}

Contact between two gears always occur for either front-front flank contact, or rear-rear flank contact. For each of the two contact cases (front or rear), there is a line of contact $(\operatorname{LoC})$, along which the contact between the teeth will be located. The front and rear contact cases are analogous, and to avoid going into details about sign conventions, only the front case will be considered from here on. Using the wheel positions, the distance between these points, $a_{\mathrm{w}}$ can be related to the angle of LoC, $\alpha_{\mathrm{w}}$. See Figure 4.

$$
a_{\mathrm{w}}=\frac{m}{2}\left(z_{1}+z_{2}\right) \frac{\cos \alpha_{0}}{\cos \alpha_{\mathrm{w}}}
$$

Here, the indices 1 and 2 mean gear wheel 1 and gear wheel 2 respectively. The LoC normal (in two 


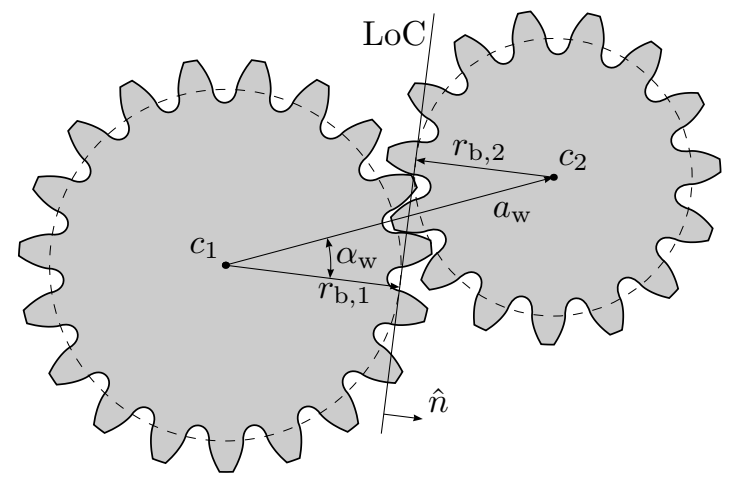

Figure 4. Line of contact of two gears, for contact between front flanks on the teeth (transmitting force when either gear is driving with positive direction of rotation). The LoC for contact between rear flanks is obtained by reflection in the line through the gear centers.

dimensions), $\hat{n}$, can then be expressed using the angle $\alpha_{\mathrm{w}}$, as

$$
\begin{gathered}
\cos \alpha_{\mathrm{w}}=\frac{\left(r_{\mathrm{b} 1}+r_{\mathrm{b} 2}\right)}{\left\|a_{\mathrm{w}}\right\|} \\
\sin \alpha_{\mathrm{w}}= \pm \sqrt{1-\cos \alpha_{\mathrm{w}}^{2}} \\
\hat{n}=\left(\begin{array}{cc}
\cos \alpha_{\mathrm{w}} & \sin \alpha_{\mathrm{w}} \\
-\sin \alpha_{\mathrm{w}} & \cos \alpha_{\mathrm{w}}
\end{array}\right) \frac{a_{\mathrm{w}}}{\left\|a_{\mathrm{w}}\right\|}
\end{gathered}
$$

where the sign of $\sin \alpha_{\mathrm{w}}$ reflects the choice between LoC for clockwise or counter-clockwise rotation.

A point $p$ on the flank of a tooth can now be described using $\alpha_{\mathrm{w}}$, the LoC, $r_{\mathrm{b}}$, and the center position of the gears, $c_{\mathrm{i}}$.

In order to determine contact forces between two gear flanks, we define an indentation depth, $\delta$ measuring the amount of intersection between the teeth. The depth is modeled using the points $p_{1}$ and $p_{2}$, where the gear flanks intersect with the LoC.

\subsection{Tip relief}

To get a smooth contact force, a modification of the tip is usually done, called a tip relief. This can be done by removing a small portion of the tooth, as shown in Figure 5. The LoC will be affected by this modification, but the change is on the scale of 0.01
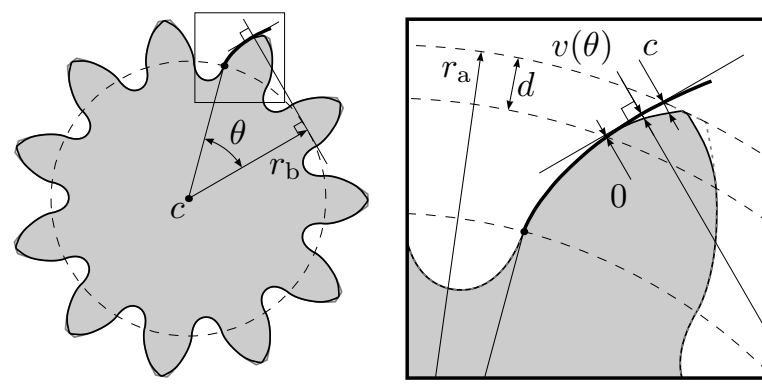

Figure 5. Gear with tip relief (exaggerated). modules, which makes the effect on the LoC negligible. The new tooth shape with tip relief can then be calculated by the following standard equation:

$$
v(\theta)=q\left(\theta-\theta_{\mathrm{a}}\right)
$$

where $q$ is a coefficient to ensure that the amplitude is obtained correctly, and $\theta_{a}$ is the maximum value for the roll angle. How much of the tooth that is removed can be specified by setting the distances $c$ and $d$, defining the tip relief amplitude and tip relief length, respectively.

\subsection{Tooth pair activation}

A pair of teeth in contact are identified by one integer index on each gear, $i_{1}$ and $i_{2}$. Together with the rotations of the axes on which the gears are mounted, $\varphi_{1}$ and $\varphi_{2}$, the directions $k_{1}$ and $k_{2}$ pointing at the starting points of the involutes on the base circles follow, which in case of front flank contact are given by

$$
\begin{array}{ll}
\hat{k}_{1}=\left(\begin{array}{c}
\cos \beta_{1} \\
\sin \beta_{1}
\end{array}\right) & \beta_{1}=\frac{s_{\mathrm{b}, 1}}{2 r_{\mathrm{b}, 1}}+i_{1} \frac{2 \pi}{z_{1}}+\varphi_{1} \\
\hat{k}_{2}=\left(\begin{array}{c}
\cos \beta_{2} \\
\sin \beta_{2}
\end{array}\right) & \beta_{2}=\frac{s_{\mathrm{b}, 2}}{2 r_{\mathrm{b}, 2}}+i_{2} \frac{2 \pi}{z_{2}}+\varphi_{1}
\end{array}
$$

As was shown in Figure 3, the rolling angles follow for any given direction of the LoC. In Figure 6, the front flanks corresponding to indices $i_{1}$ and $i_{2}$ are marked with a thick line. As the gears rotate, the current tooth pair will become disengaged, while other pairs will become engaged. An index skip, $i_{\Delta}$, is chosen by upward rounding of the gear contact ratio. Depending on the direction of rotation, the next tooth pair to follow when the current pair has become disengaged is selected as

$$
\begin{aligned}
& i_{1}^{\prime}=i_{1} \pm i_{\Delta} \\
& i_{2}^{\prime}=i_{2} \mp i_{\Delta}
\end{aligned}
$$

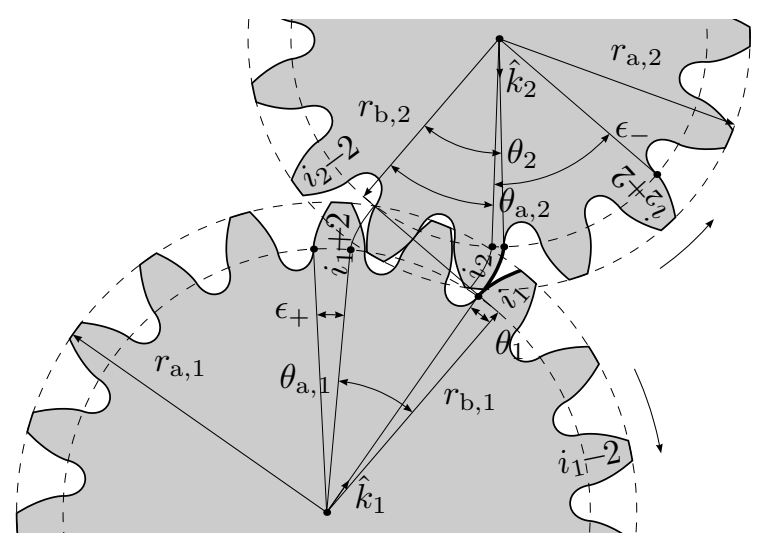

Figure 6. Slack variables used to control tooth pair activation for front flank contact. Here, $i_{\Delta}=2$, corresponding to a configuration for a contact ratio between 1 and 2 . 
In Figure 6 where gear 1 rotates clockwise, the change in $i_{1}$ is with positive sign, while the change in $i_{2}$ is with negative sign. The slack angle $\epsilon_{+}$measures how much the rolling angle $\theta_{1}$ may decrease until it is absolutely necessary to consider interaction for the tooth pair $i_{1}^{\prime}$ and $i_{2}^{\prime}$. Analogously, when gear 2 rotates clockwise, $\epsilon_{-}$measures how much the rolling angle $\theta_{2}$ may decrease until it is absolutely necessary to consider interaction for the tooth pair in the opposite direction. The slack angles are given by

$$
\begin{aligned}
& \epsilon_{+}=\theta_{1}+i_{\Delta} \frac{2 \pi}{z_{1}}-\theta_{\mathrm{a}, 1} \\
& \epsilon_{-}=\theta_{2}+i_{\Delta} \frac{2 \pi}{z_{2}}-\theta_{\mathrm{a}, 2}
\end{aligned}
$$

In the Modelica model presented in Section 4, the tooth indices are updated such that the slack angles are positive at all times. For robust simulation, it is desirable to update indices with some margin until it is absolutely necessary according to the slack variables. That is, we should avoid triggering the update at the lower bound 0 . When indices are updated because one of the slacks is getting too close to zero, that slack variable will be reset to a large value, while the other slack variable will be signifiantly reduced. We will trigger based on the following conditions, where $\gamma$ is a positive constant which remains to be determined,

$\epsilon_{+}<\gamma\left(\epsilon_{-}-i_{\Delta} \frac{2 \pi}{z_{2}}\right) \quad$ Trigger positive change in $i_{1}$

$\epsilon_{-}<\gamma\left(\epsilon_{+}-i_{\Delta} \frac{2 \pi}{z_{1}}\right) \quad$ Trigger negative change in $i_{1}$

In order to avoid endless event iteration when updating the indices, it must be ensured that triggering a change in one direction does not reduce the other slack so much that it satisfies the condition for re-triggering a change in the opposite direction. By equating the margin to the lower bound of zero slack, with the margin to re-triggering a change in the opposite direction, a natural choice of $\gamma=\frac{\sqrt{5}-1}{2}$ is obtained.

\section{Hertz Contact Stress}

To calculate the force between two gear teeth, Hertzian Contact Theory has been used. At the point of contact, the two teeth are approximated by two cylinders with parallel axes, see Figure 7.

The indentation depth, $\delta$, is related to the contact force by

$$
\delta=\frac{2 F\left(1-\nu^{2}\right)\left(\frac{2}{3}+\log \left(\frac{4 R_{1}}{b}\right)+\log \left(\frac{4 R_{2}}{b}\right)\right)}{\pi L E_{\bmod }}
$$

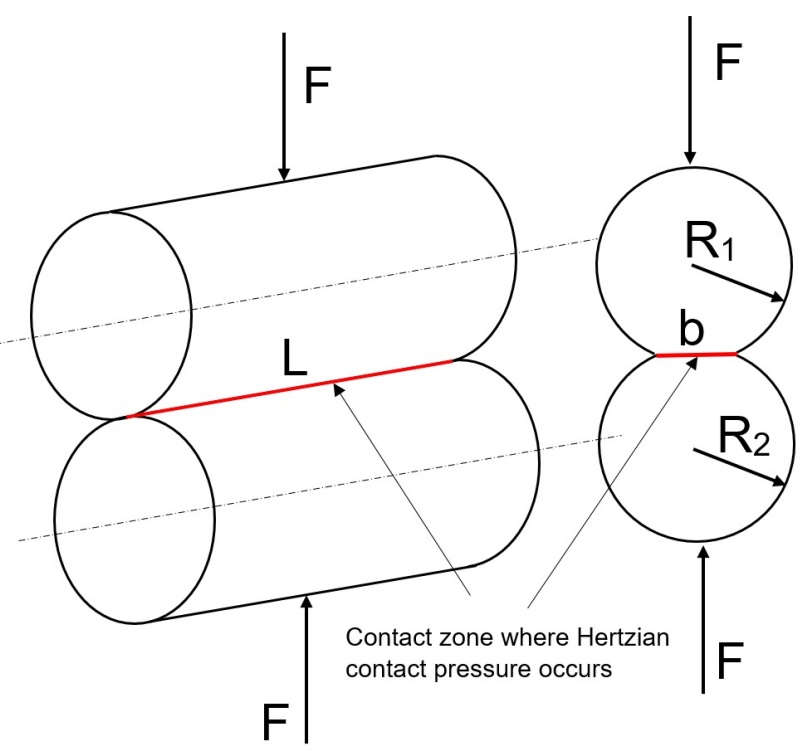

Figure 7. Contact between two cylinders with variables.

where $F$ is the force, $\nu$ is the Poisson ratio, $E_{\text {mod }}$ is Young's modulus, $L$ is the length of the cylinder, $R_{i}$ are the radii of the cylinders, and $b$ is the contact width $^{2}$. The contact width is modeled by

$$
b=\sqrt{\frac{32 F}{\pi L E_{\text {red }}\left(\frac{1}{R_{1}}+\frac{1}{R_{2}}\right)}}
$$

Here, $E_{\text {red }}$ is a combination of the two gear wheels' material parameters

$$
\frac{1}{E_{\mathrm{red}}}=\frac{1}{2}\left(\frac{1-\nu_{1}^{2}}{E_{\bmod 1}}+\frac{1-\nu_{2}^{2}}{E_{\mathrm{mod} 2}}\right)
$$

From (17) and (18), the force can be calculated as a function of the indentation depth. The geometry of the cylinders in contact will change when moving on the LoC. This means that the curvature radii $R_{1}$ and $R_{2}$ will be changing so that one of them will start with a small radius and increase when moving along the $L o C$, and the other will start with a big radius and decrease when moving along the $L o C$.

The conditions for applying a force at the appropriate time is to check if the distance between the wheel center $c_{\mathrm{i}}$ and the point $p_{\mathrm{i}}$ is less than the top radius of the gear $r_{\mathrm{a}, i}$. Additionally, we also check if $\delta$ is larger than zero, i.e.

$$
\begin{aligned}
\left|c_{1}-p_{1}\right| & <r_{\mathrm{a}, 1} \\
\left|c_{2}-p_{2}\right| & <r_{\mathrm{a}, 2} \\
\delta & >0
\end{aligned}
$$

The effects of gear damping has not been included in this model.

\footnotetext{
${ }^{2}$ As found in Roark's Formulas for Stress and Strain, 2002, Table 14.1.2
} 


\section{Modelica Model}

The implementation of the spur gear model in Modelica is using the Modelica MultiBody library. This means that there will be 3D animations for all models. For example, the contact force is visualized by arrows between two teeth, as seen in Figure 8 .

The topology of the model consists of a top layer diagram where the layout is specified, i.e. how the gear wheels are positioned and if a force should be calculated between them. Figure 9 shows the Modelica diagram layer of a simple two wheel model with contact between them.

The force between the two gear wheels is calculated inside the gearForceCalculation component. The system of non-linear equations (17) and (18) has multiple solutions, and the correct one is not

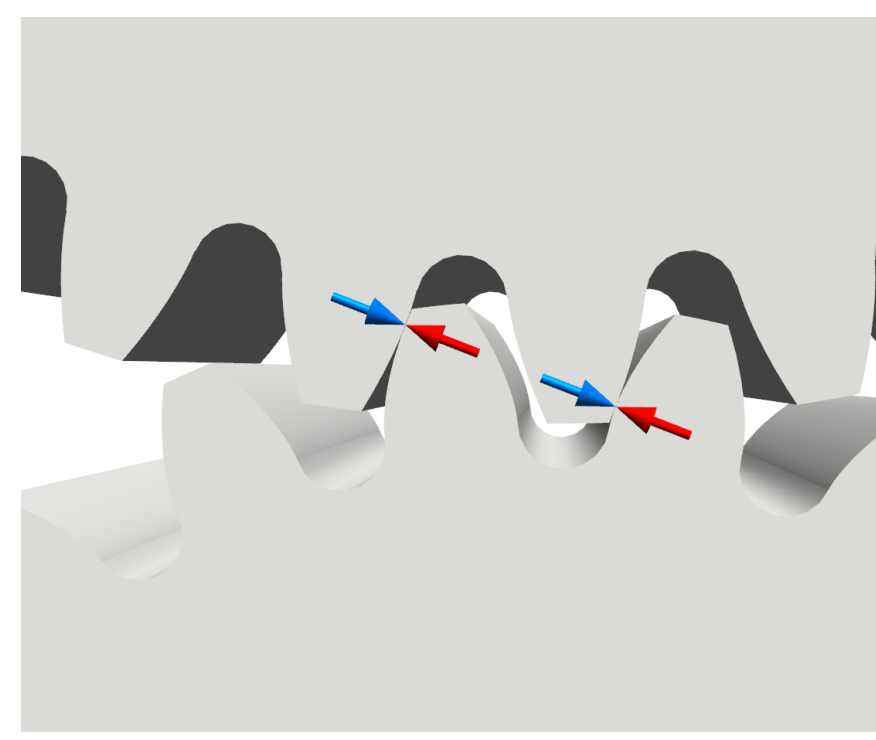

Figure 8. Two outer spur gears in contact, with arrows representing the contact forces acting on the gears. At this moment, two teeth pairs are in contact.

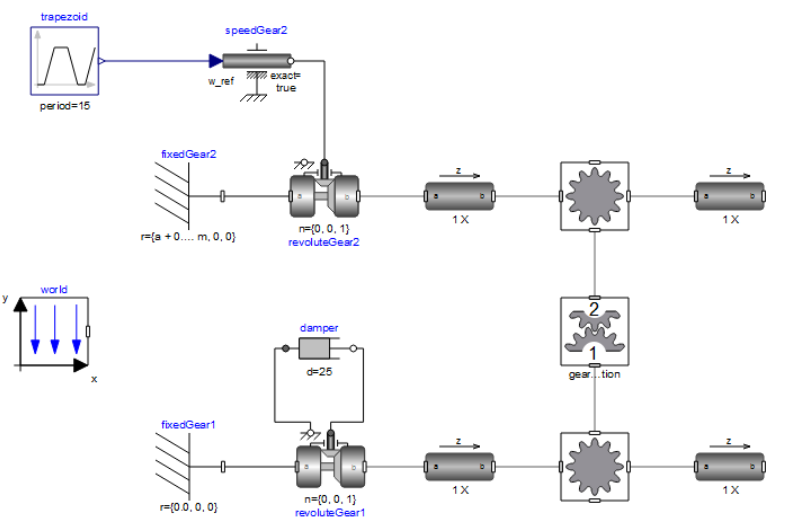

Figure 9. Model of two gear wheels in contact.

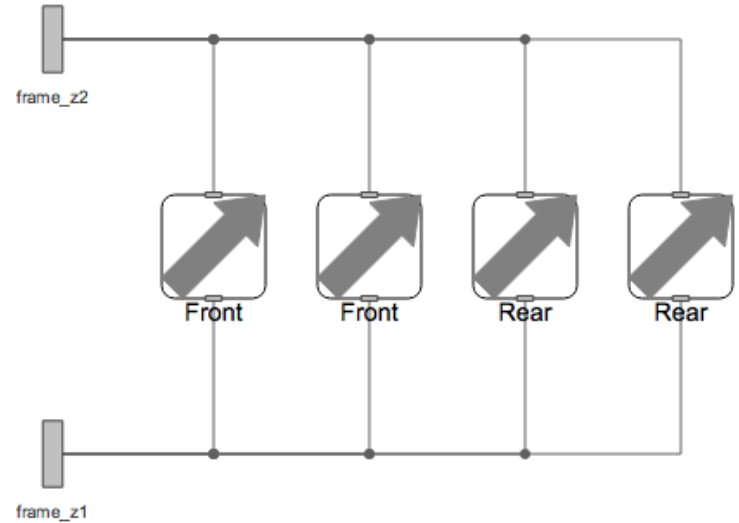

Figure 10. Model of the component where gear forces are calculated, containing four ContactForcePoints.

differentiable at $\delta=0$. To handle this, the solution is approximated by a closed-form expression. The gearForceCalculation class contains at least four ContactForcePoint components, as seen in Figure 10. Each of these ContactForcePoints is responsible for calculating one force pair on one matching teeth pair. Since the gear ratio should be above one, but less than two in the case of two outer spur gears, two force pairs are needed. Two more force pairs (ContactForcePoint components) are needed due to the two flanks on each tooth. If a gear ratio over 2 is possible, as in the case of a planetary gear between the ring and a planet, more ContactForcePoints can be added to handle this.

The contact ratio will be calculated automatically, depending on the geometry of the two gear wheels in contact. This is used to assert that the GearForceCalculation component is set up correctly.

Many parameters can be set by set user on the GearForceCalculation component that will affect the ContactForcePoints inside. The parameters are listed in Table 2.

Table 2. GearForceCalculation parameters.

\begin{tabular}{ll}
\hline Parameter & Description \\
\hline$m$ & Gear module \\
$L$ & Contact width of wheels \\
$z_{i}$ & Number of teeth in gear wheel $i$ \\
$x_{i}$ & Profile shift factor for gear wheel $i$ \\
$\nu_{i}$ & Poisson's ratio for wheel $i$ \\
$E_{\text {mod }}$ & Young's Modulus wheel $i$ \\
$c_{i}$ & Tip relief amplitude for wheel $i$ \\
$d_{i}$ & Tip relief length for wheel $i$ \\
\hline
\end{tabular}




\section{Example Models}

Figure 9 showed a simple example with two outer gears in contact, and no other components in the system. This can easily be expanded.

\subsection{Effect of tip relief}

Using the presented gear model, it is possible to compute dynamic effects in gears such as the variation in contact stresses. Figure 11 shows the contact stress for one tooth during a contact. In this case the gear wheel support is fixed in all translational directions, which means that there are no external vibrations affecting the result.

The contact starts with a transient, stabilizing to a lower force level, where two pairs of teeth in contact. Then the other pair of teeth goes out of contact and only the current tooth takes all force. After a while, a new teeth pair will go into contact, and the force level will drop again. Finally the current teeth pair goes out of contact, and the force drops to zero. The reason for the transient is that all pair of teeth in contact will have the same indentation depth, $\delta$. Hence, when a new pair of teeth goes into contact, the initial indentation depth at the tip of the incoming tooth will be that of the one pair of teeth currently in contact. The gears will then quickly adjust to the same total torque of two pairs of teeth. To avoid the transient, the tip of the involute shape is modified with a tip relief, as was shown in Figure 5.

Figure 12 shows the much smoother contact pressure with tip relief.

\subsection{Wind turbine gear box}

A planetary gear box can be created by combining inner and outer spur gears with flexible shafts. A two-stage gear box is then connected to the planetary gear box. A screenshot of the animation is shown in Figure 13 and the model diagram is shown in Figure 14. This setup is capable of changing the angular velocity from the slow rotation of the blades, around

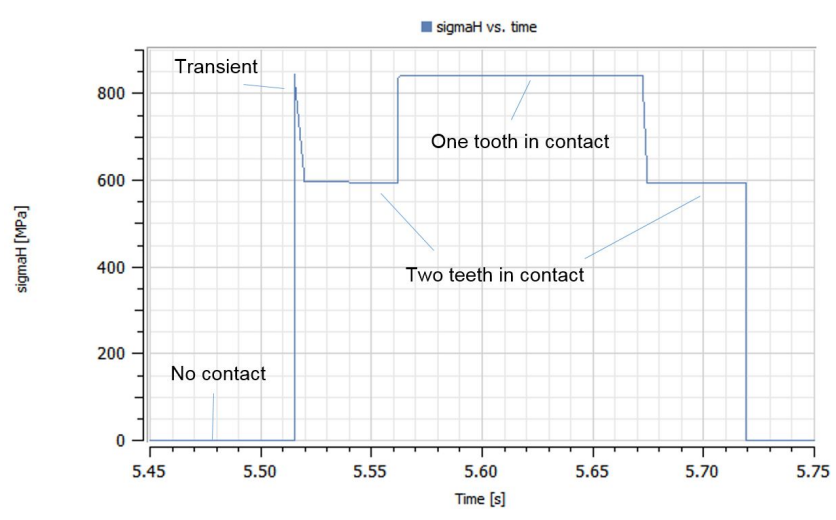

Figure 11. Contact pressure between two teeth without tip relief.

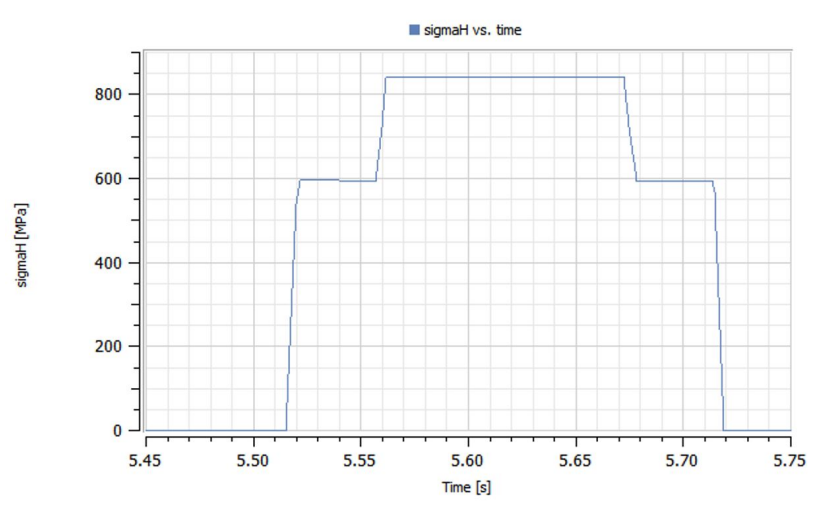

Figure 12. Contact pressure between two teeth with tip relief.

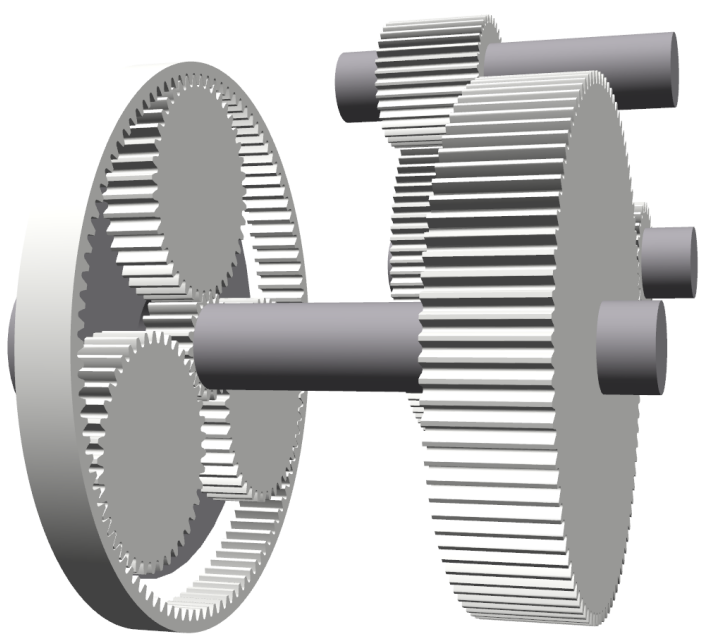

Figure 13. Animation of the wind turbine gear box model.

$10 \mathrm{rpm}$, to the fast rotation of the generator, around $1500 \mathrm{rpm}$.

The contact forces are calculated at many different points in this system. In the planetary gear there are forces between the center gear wheel (the sun) and the three outer wheels (the planets), and also between the three planets and the inner gear wheel (the ring). With a possible contact ratio above 2 , the GearForceCalculation components between inner and outer gears (planet and ring) contain 6

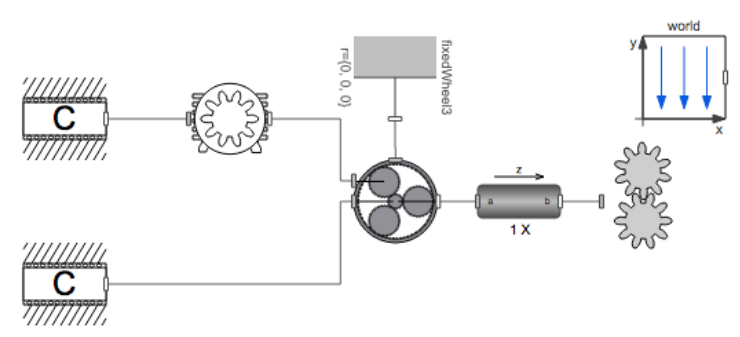

Figure 14. A wind turbine gear box. 
ContactForcePoints. In total, the system contains 38 ContactForcePoint components, making it possible to simulate this system with gear forces at all points and all rotational directions. The gear can be driven by any wheel, both clockwise and counterclockwise. In addition to this, the wheels are connected to flexible shafts that are fixed in a support.

In this setup, one could detect if there are any peaks in forces between the gears, or if the flexible shafts affect the gear in any significant way if the material parameters for the beams changes. An example of contact pressures in a steady state of the gear box can be seen in Figure 17 on page 9.

\subsection{Gear and bearings}

Since rotating machinery elements affect each other in various ways, it is important to be able to study different elements together. An example of such and interaction is when a system containing rotating flexible shafts with bearings, that sets the gear wheels in motion. The bearings contain cylindrical rollers, which have Hertzian contact forces between the inner and outer ring of the bearing. A $3 \mathrm{D}$ visualization of the setup is shown in Figure 15.

Two flexible shafts are supported by roller bearings. The bearings on the lower shaft are mounted on flexible supports, that are fixed to the ground. The lower shaft rotates at $600 \mathrm{rpm}$. One of the bearings has an outer ring defect (top right bearing in figure 15). The visualization of the outer ring in this bearing has been removed to give a better view of the rollers inside the outer wheel. This defect will in this case

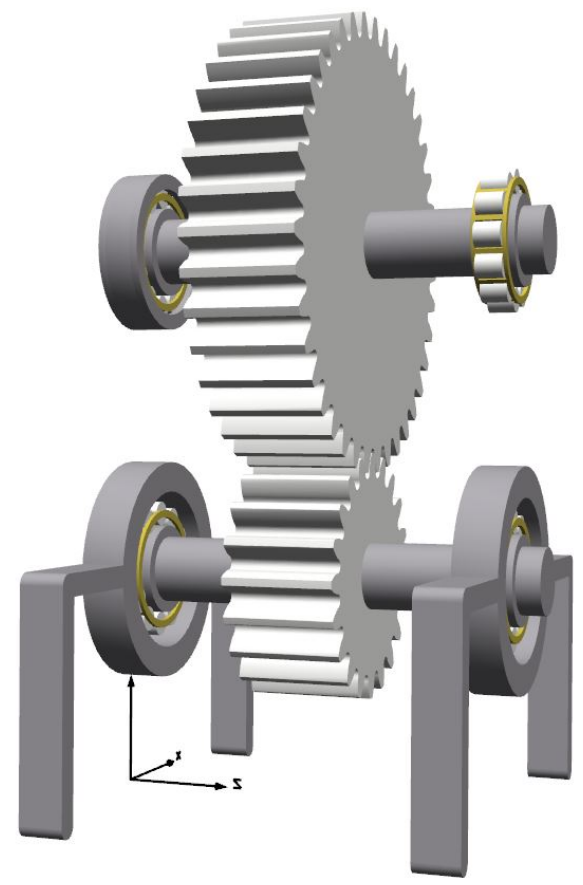

Figure 15. Two shafts with bearings, connected by spur gears.

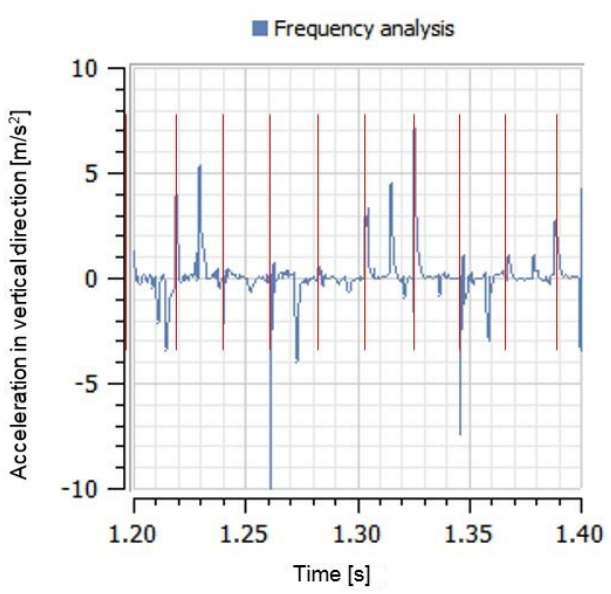

Figure 16. Acceleration in the vertical direction, with a red highlighting at impact points, as predicted by the shock pulse method.

cause an extra downward force to be applied at the "12 o'clock" position when a roller passes this point.

Vibration analysis of a damaged bearing is usually a quite complicated task. A frequency spectrum will normally not show a small bearing defect. Instead different kinds of shock pulse methods have been developed. The signals are normally at rather high frequencies. In this example the time of impact has been marked with red lines. As can be seen in figure 16, the accelerations and impacts align at most points. This analysis can also be done to investigate a damaged gear.

The benefit of being able to do this simulation analysis is huge. Understanding where and how large a damage is gives a better picture of what and when an overhaul should be done, often saving a lot of money as well as improving the overhaul. A typical example of where this is vital is in paper machines where a carefully planned overhaul may save millions of dollars. Another example is in cruise ship machines, where a dry-dock needs to be available for an overhaul and passengers might need to rebook their trip to other ships, depending on overhaul time. 


\section{Conclusions}

With this implementation of a gear model, a high accuracy causal model of contact forces can be put in a multi-domain simulation environment. This can then be used to find weak points in complex rotating machinery systems. Key points are summarized below.

- The physical accuracy is at the level where factors like shaft position, clearances, tip relief, misalignment and vibrations by default are taken into account. Essentially, this approach combines the simplicity of drag-and-drop, and multidomain modeling in Modelica, as in (van der Linden, 2012), with high accuracy calculations of gears, as in (Kosenko and Gusev, 2012).

- The drag-and-drop capability of Modelica and Wolfram SystemModeler, makes the creation of a custom model very easy. All effort required from a user is to parametrize the models.

- The formulation of the gear contact shown in this paper is designed to be simple to expand into more complex contacts. In other words it is easy to take deviations from the ideal involute gear into account. Tip relief was used here as an example. Another effect of this is that the gear formulation can also easily be expanded to helical gears, bevel gears and worm gears. Effects such as contact roughness, and manufacturing errors can also be included in the future.

- Domain specific software, such as gear or bearing design software, gives very accurate results for a specific machine element, but are limited when an extension outside the domain is needed. The examples presented in this paper shows how efficient several different machine elements can be combined, as well as coupling to other domains. For instance, the applied torque in the models was obtained using a PID controller.

- The wind turbine example showed how important tip relief is for avoiding excessive wear. Using a software not able to include tip relief may lead to a bad geometrical design and high costs in the correction process. This is particularly true if the wear is detected after some time in operation.

- What is missing and can be included in future work, is testing and verification of the modeling results as done in (van der Linden, 2015), as well as a speed up of the simulation time. Today, the simulation time is around real-time for simple models ( 2 spur gears in contact, both connected on flexible shafts), and slower for more complex models, depending on speed of rotation. Tooth bending has been neglected in this model and should be included in the future

\section{References}

Ivan Kosenko and Il'ya Gusev. Implementation of the spur involute gear model on modelica. Proceedings of the 8th International Modelica Conference, 2011. URL https://www.modelica.org/events/modelica2011/ Proceedings/pages/papers/13_3_ID_117_a_fv.pdf.

Ivan Kosenko and Ilya Gusev. Revised and improved implementation of the spur involute gear dynamical model. Proceedings of the 9th International Modelica Conference, 2012. doi:10.3384/ecp12076311.

M. Otter, M. Dempsey, and C. Schegel. Package PowerTrain: A Modelica library for modeling and simulation of vehicle power trains. Modelica Workshop 2000 Proceedings, pages 22-32, 2000. URL https://www .modelica.org/events/workshop2000/ index_html/proceedings/Otter.pdf.

F.L.J. van der Linden. Modelling of elastic gearboxes using a generalized gear contact model. Proceedings of the 9 International Modelica Conference, 2012. doi:10.3384/ecp12076303.

F.L.J. van der Linden. Modeling of geared positioning systems: An object-oriented gear contact model with validation. Proceedings of the Institution of Mechanical Engineers, 2015. doi:10.1177/0954406215592056.

F.L.J. van der Linden and P.H. Vazques de Souza Silva. Modelling and simulating the efficiency and elasticity of gearboxes. Proceedings 7th Modelica Conference, 2009. doi:10.3384/ecp09430052.

Dirk Zimmer. A planar mechanical library for teaching modelica. Proceedings of the 9th International Modelica Conference, 2012. doi:10.3384/ecp12076681. 

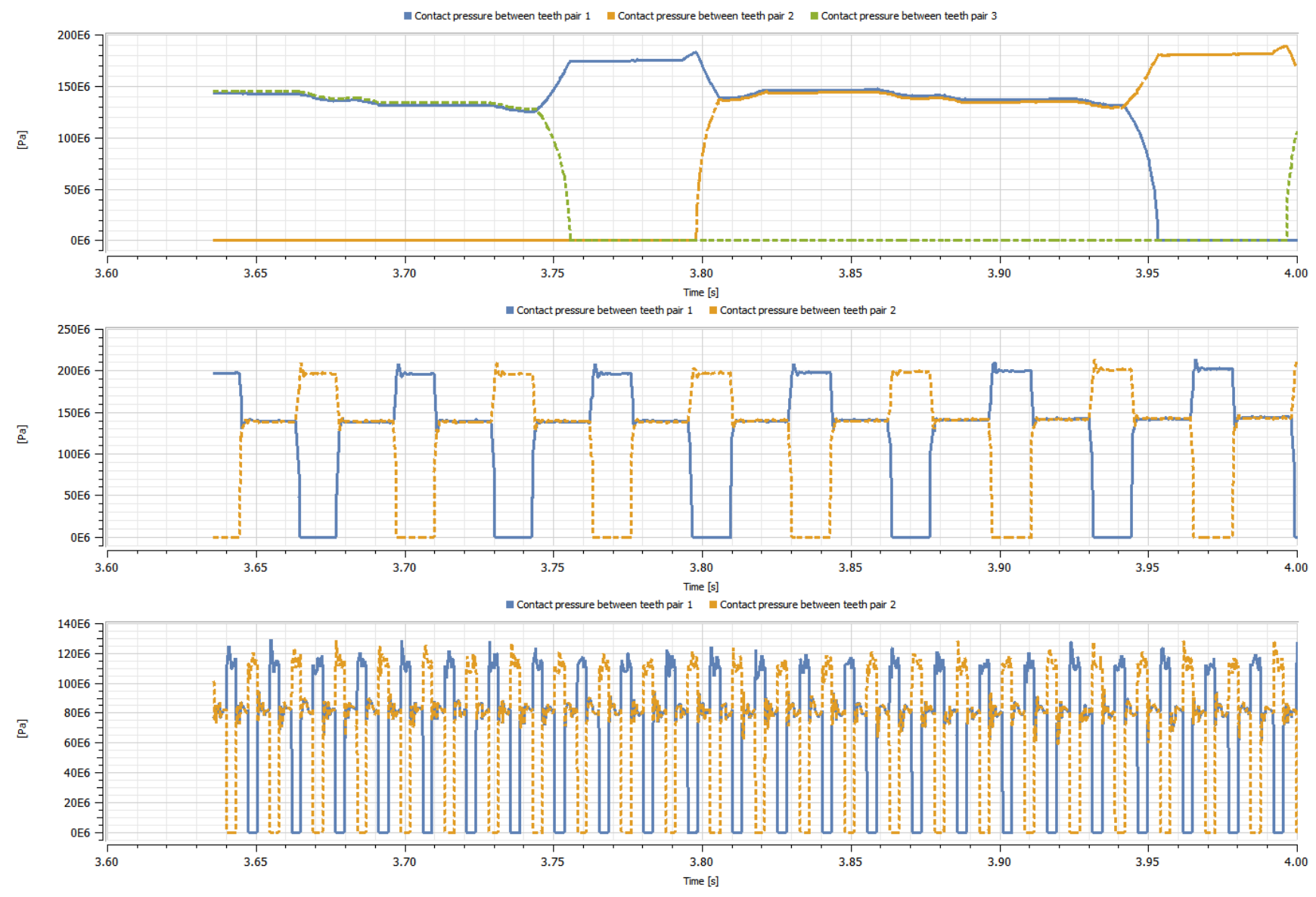

Figure 17. Contact pressure on teeth at three places in the wind turbine gear box. The first plot is showing the pressure of one planetary wheel to the ring. The second plot shows the pressure between teeth in the first step in the two-step gear box. Finally the third plot shows the pressure between teeth in the last step of the gear box. One can easily see how the frequency changes from the planetary gear to the last step before the generator. 